\title{
Dosimetric and Mechanical Stability of CyberKnife Robotic Radiosurgery Unit: 5 Years' Clinical Experience
}

\author{
Fatih BíLTEKIN, Gözde YAZICl, Mustafa CENGiZ, Ali DOĞAN, \\ Bülent ÜNLÜ, Mete YEĞiNER, Gökhan ÖZYiĞiT \\ Department of Radiation Oncology, Hacettepe University Faculty of Medicine, Ankara-Turkey
}

\begin{abstract}
OBJECTIVE
The purpose of the present study was to investigate dosimetric and mechanical stability of CyberKnife Robotic Radiosurgery System (Accuray Inc., Sunnyvale, CA, USA) in short- and longterm period.

\section{METHODS}

Output factor measurements and automated quality assurance (AQA) tests performed on CyberKnife unit 2009-2013 at radiation oncology department of Hacettepe University, Turkey, were analyzed retrospectively.
\end{abstract}

\section{RESULTS}

According to the analysis, more than $95 \%$ of the output measurements over 5 years were within the tolerance limit $\leq 2 \%$. In AQA test analysis, $144 \mathrm{AQA}$ test results were within the tolerance limit from 2009 to 2011. However, 7 of the 51 measurements taken in 2012, and 4 of the 47 measurements performed in 2013 exceeded $1 \mathrm{~mm}$ radial error.

\section{CONCLUSION}

Output and AQA data of CyberKnife system indicate that it is quite stable in daily and long-term period. Nevertheless, daily measurements should be performed on CyberKnife unit since high radiation dose per fraction is usually delivered to target volume.

Keywords: CyberKnife unit; output stability; stereotactic radiosurgery.

Copyright $\odot$ 2016, Turkish Society for Radiation Oncology

\section{Introduction}

The main purpose of radiotherapy (RT) is to deliver an accurate absorbed dose to the target volume while staying within acceptable tolerance limits for surrounding normal tissue and critical organs in order to minimize collateral effects. Over the last 2 decades, many improvements have occurred in the field of radiation therapy such as new treatment modalities (intensity-modulated radiotherapy [IMRT], volumetric-modulated arc therapy [VMAT] and stereotactic radiosurgery/radiotherapy [SRS/SRT]), new image-guided systems (in-room computed tomography [CT] techniques, magnetic resonance imaging [MRI]-guided RT and ultrasound [US]based systems), as well as new quality assurance (QA) and quality control (QC) systems. Nevertheless, there 
are still inevitable uncertainties due to the dosimetric and mechanical stability of the linear accelerator (linac) over a period of time. Therefore, efficient QC programs should be put in place to minimize uncertainty based on machine characteristics and parameters.

Dosimetric and mechanical QC of a linac is the process of keeping the accuracy of machine functions within suggested tolerance limits.[1,2] Reports and documents for these suggested values have been published by the International Atomic Energy Agency (IAEA), the American Association of Physicists in Medicine (AAPM), the European Society for Therapeutic Radiology and Oncology (ESTRO), and by several other national and international organizations. [3] The main purpose of all these QA programs is to standardize function of the treatment and measurement equipment used in RT facilities, and thus to maximize quality of patient care. The times tables and tolerance limits of QC for conventional linacs and SRS/ SRT units have been studied widely in the literature. [2,4-10] Moreover, control charts, as used in industrial manufacturing and other healthcare systems, have recently been applied to QA for RT to determine shortand long-term stability of conventional linacs.[11-22] However, there does not appear to be a study analyzing short- and long-term output stability of CyberKnife Robotic Radiosurgery System (Accuray Inc., Sunnyvale, CA, USA).

In SRS/SRT facilities with CyberKnife, high radiation dose is usually delivered to target volume with sub-millimeter accuracy. Therefore, both mechanical accuracy and dosimetric stability of the system play important roles in the precision of the treatment. [4] The present study is an analysis of the short- and long-term output and target positioning stability of $\mathrm{Cy}$ berKnife unit.

\section{Materials and Methods}

\section{Output stability analysis for CyberKnife Robotic Radiosurgery System}

Output measurements collected by department of radiation oncology at Hacettepe University, Turkey, between 2009 and 2013 were analyzed to evaluate the short- and long-term output stability of G4 CyberKnife Robotic Radiosurgery unit. Output was calibrated to $100 \mathrm{cGy}=100 \mathrm{MU}$ (or $1 \mathrm{cGy}=1 \mathrm{MU}$ ) at $1.5 \mathrm{~cm}$ depth in water using AAPM Task Group (TG) 51 protocol[23] for reference dose calibration as defined in Table 1 . In 5 years, measurements were performed on daily basis
976 times using $0.6 \mathrm{cc}$ PTW Farmer ionization chamber (PTW, Freiburg Germany) with buildup cap attached to the birdcage chamber holder, using necessary correction factors according to AAPM TG-51 protocol (Eq. 1).[23] Measurements were repeated 3 times in every daily setup and average value was taken as output value. When the difference between measured output and reference value exceeded threshold of $2 \%$, output calibration was usually performed before starting the treatment. Readings before and after calibration were recorded in output database.

Equation for the dose absorbed in water for radiation of quality $\mathrm{Q}$ is

$D_{W}^{Q}=M N_{D, w}^{Q}$

$D_{W}^{Q}$ : Dose in water,

M: Corrected electrometer reading

$M: \mathrm{P}_{\text {ion }} \mathrm{P}_{\text {TP }} \mathrm{P}_{\text {elec }} \mathrm{P}_{\text {pol }} \mathrm{M}_{\text {raw }}$

$\mathbf{P}_{\text {ion }}$ : Correction for incomplete ion collection efficiency

$\mathbf{P}_{\mathrm{TP}}$ : Temperature-pressure correction, which adjusts reading to standard environmental conditions

$\mathbf{P}_{\text {elec }}$ : Correction for electrometer's calibration factor if electrometer and ion chamber are calibrated separately

$\mathbf{P}_{\text {pol }}$ : Correction for any polarity effects

$M_{\text {raw }}$ : Raw ion chamber reading

$N_{D, w}^{Q}$ : Absorbed-dose to water calibration factor for $D, w$ an ion chamber

\section{AQA test analysis for CyberKnife Robotic Radiosurgery System}

The AQA test is an isocentric targeting accuracy or robot pointing test to verify delivery accuracy of $\mathrm{Cy}$ berKnife unit; it is similar to the Winston-Lutz test[24] commonly used in gantry-based SRS/SRT systems. In the present study, 242 AQA tests (from 2009-2013) performed on CyberKnife system at Hacettepe University were analyzed retrospectively. Custom designed AQA phantom containing $2 \mathrm{~cm}$ tungsten ball hidden in cubic phantom was used for the measurements (Figure 1). In the QA, 2 orthogonal gafchromic films were placed inside the phantom (Figure 1) and an isocentric treatment consisting of 2 beams in anterior/posterior and lateral directions were delivered tracking 4 fiducial markers (Figure 2). Targeting error (Eq. 2) was determined by analyzing the offset from the center of concentric circles formed by the shadow of the metal ball 
Table 1 Reference conditions for output measurement in Cyberknife unit

\begin{tabular}{ll} 
Influence quantity & Reference value or reference characteristics \\
\hline Phantom material & Water (in daily measurements buildup cap is used with correction factor) \\
Chamber type & Cylindrical \\
Measurement depth $z_{\text {ref }}$ & $1.5 \mathrm{~g} / \mathrm{cm}^{2}$ \\
Reference point of the chamber & On the central axis at the center of cavity volume \\
Position of the reference point of the chamber & At the measurement depth $z_{\text {ref }}$ \\
SSD/SCD & $80 \mathrm{~cm}$ \\
Collimator size & $60 \mathrm{~mm}$ in diameter \\
\hline
\end{tabular}

and the acrylic target sphere in anterior/posterior, left/ right and superior/inferior directions. If radial error deviated more than $1 \mathrm{~mm}$ from reference value, endto-end (E2E) tests were performed and the manipulator was recalibrated. All these data were recorded in AQA database.

Targeting error $=\sqrt{\left(\text { offset }_{\text {sup-ing }}\right)+\left(\text { offset }_{\text {left-right }}\right)+\left(\text { offset }_{\text {ant-post }}\right)}$

\section{Results}

\section{Output stability analysis for CyberKnife Robotic Radiosurgery System}

Daily output data of the CyberKnife system is shown

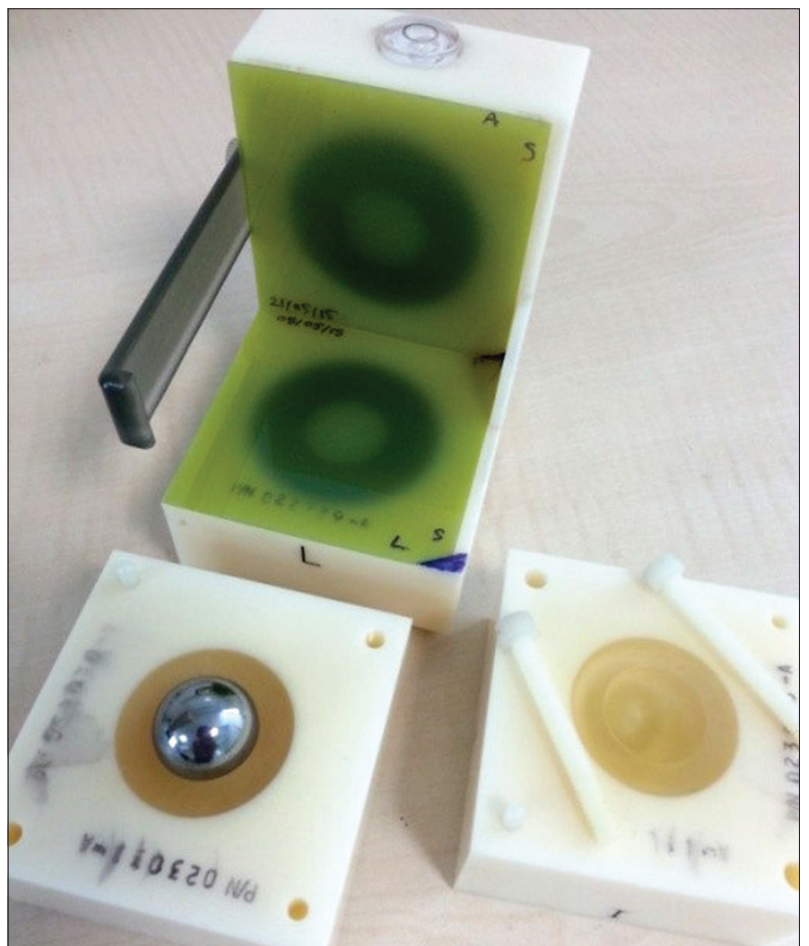

Fig. 1. AQA phantom with 2 orthogonal films after exposure. in Figure 3. In 2 of 976 measurements, differences with respect to reference value of $100 \mathrm{cGy}$ were more than $3 \%$. However, over 5 years, more than $95 \%$ of measurements were within tolerance limits $(\leq 2 \%)$. Although percentage of measurements exceeding the limit was nearly $5 \%$, dose calibration was performed in only $2 \%$ of the measurements. If limits were exceeded due to a change in ambient conditions, such as room temperature and pressure, calibration was not performed until treatment of first patient, and additional measurements were taken prior to every treatment until stabilization of environment conditions. According to analysis of yearly measurements over 5 years (Figure 3 ), output of CyberKnife system is quite stable in long-term period. In addition to variation of output trend over time as illustrated in Figure 3, statistical analyses of output values on yearly basis were evaluated, and are presented in Table 2.

\section{AQA Test Analysis for CyberKnife Robotic Radiosurgery System}

Between 2009-2011, 144 AQA tests performed on Cy-

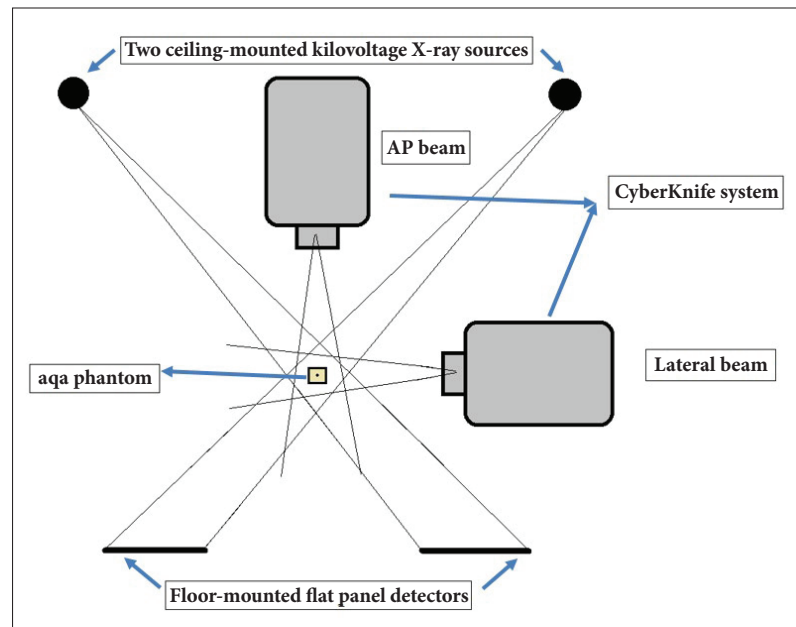

Fig. 2. AQA test geometry in CyberKnife unit. 
Table 2 Statistical analysis of output stability

\begin{tabular}{|c|c|c|c|c|c|}
\hline Statistical analysis & 2009 & 2010 & 2011 & 2012 & 2013 \\
\hline Mean value $(\mathrm{cGy})^{*}$ & 100.25 & 100.01 & 99.77 & 99.72 & 99.74 \\
\hline Standard deviation (cGy) & 1.08 & 1.38 & 0.98 & 1.021 & 0.82 \\
\hline Median value $(\mathrm{cGy})^{*}$ & 100.5 & 100.0 & 99.7 & 99.8 & 99.8 \\
\hline Maximum value (cGy)* & 102.4 & 102.9 & 102.5 & 102.8 & 102.9 \\
\hline Minimum value $(\mathrm{cGy})^{*}$ & 96.7 & 96.6 & 97.59 & 97.6 & 98.1 \\
\hline \# of measurement & 190 & 209 & 242 & 195 & 140 \\
\hline \# of recalibration & 4 & 7 & 5 & 2 & 1 \\
\hline
\end{tabular}

*Reference dose: 100 cGy

Table 3 Yearly basis statistical analysis of targeting error of CyberKnife system

\begin{tabular}{lccccc} 
Statistical analysis & 2009 & 2010 & 2011 & 2012 & 2013 \\
\hline Mean value $(\mathrm{mm})$ & 0.396 & 0.358 & 0.467 & 0.776 & 0.847 \\
Standard deviation $(\mathrm{mm})$ & 0.176 & 0.222 & 0.156 & 0.321 & 0.337 \\
Maximum radial error $(\mathrm{mm})$ & 0.944 & 0.809 & 0.865 & 1.428 & 1.483 \\
No. of measurements exceeding tolerance limit & 0 & 0 & 0 & $7^{* *}$ & $4^{* * *}$ \\
No. of recalibrations & 0 & 0 & 0 & $1^{* *}$ \\
\hline
\end{tabular}

*Tolerance limit: radial error $<1 \mathrm{~mm}$.

**7 measurements exceeding tolerance limit was performed in May 2012 and manipulator was recalibrated after end-to-end (E2E) test analysis.

***4 measurements exceeding tolerance limit was performed in November 2013 and manipulator was recalibrated after E2E test analysis.

berKnife unit were found to be within the tolerance limit of a radial error of $<1 \mathrm{~mm}$. Although 11 of the 98 measurements taken during 2012 and 2013 exceeded the tolerance limit, 7 of 11 measurements were from May 2012 and 4 were taken in November 2013. The manipulator was recalibrated 2 times over 5 years. It can be seen in Table 3 that there is an increasing trend in radial error from 2010 to 2013 . Nevertheless, yearly mean radial error variation between consequent years was less than $0.4 \mathrm{~mm}$ and maximum targeting error was also less than $1.5 \mathrm{~mm}$ over the 5-year period. The present analysis indicates that targeting precision of CyberKnife system on annual basis was under $1 \mathrm{~mm}$ (Table 3).

\section{Discussion}

In the literature, there are various studies and suggestions based on long-term clinical experience about the process for and frequency of routine $\mathrm{QC}$ checks as well as stability analysis of conventional linacs. Although the working principle and safety aspects of CyberKnife system are quite different from other X-ray based accelerators, there is a limited number of studies about QA procedure and stability of CyberKnife unit with respect to the conventional linacs. Therefore, in the pres- ent study, we analyzed the short- and long-term output stability and targeting accuracy of CyberKnife robotic radiosurgery system.

The study revealed that output of CyberKnife unit was quite stable based on daily and annual measurements (Figure 3). Almost 5\% of measurements exceeded tolerance limits in 5 years. According to our

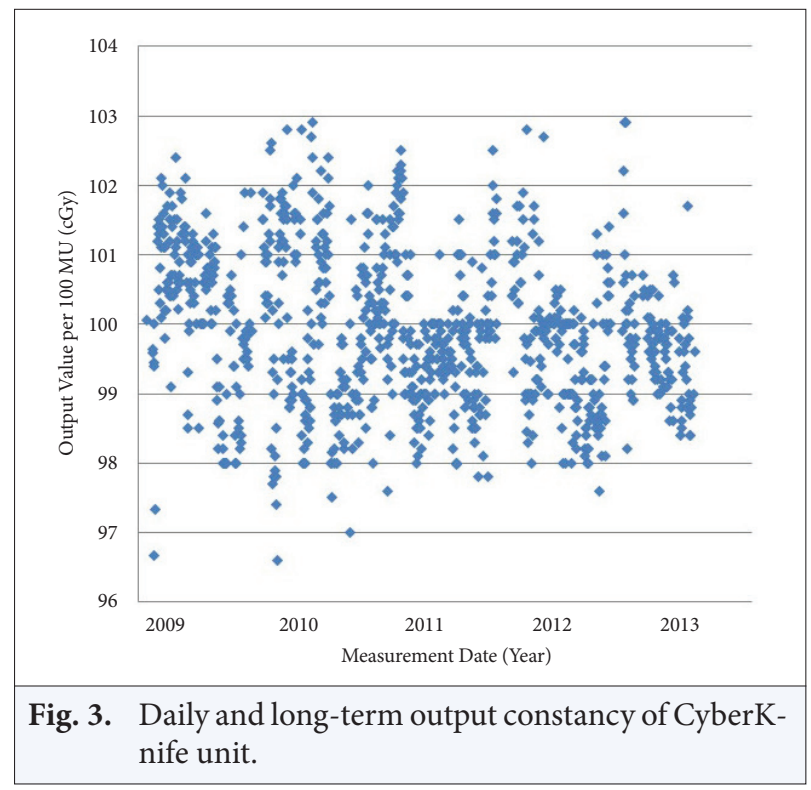


measurement database, a change in environmental conditions had the highest contribution to this variation. Sharma et al.[25] also pointed out that since the CyberKnife uses ion chamber vented to the atmosphere, changes in ambient conditions like temperature and pressure can cause deviation of output value from the baseline. Therefore, output measurements should be performed at least daily, and measurements should be repeated prior to every patient if the ambient conditions change dramatically. In addition to environmental conditions, according to IAEA code of practice TRS-398,[26] user's beam-based factors such as long-term stability of user dosimeter, establishment of measurement setup, dosimeter reading relative to beam monitor, correction for influence quantities and for beam quality cause an uncertainty of greater than $1 \%$ in the measurement of absorbed dose to water in reference depth. Therefore, all of these uncertainties should be taken into consideration and several precautions should be taken to minimize the beam-based uncertainty. As a precaution, all measurements should be performed by a licensed medical physicist/medical dosimetrist or a qualified RT technician knowledgeable about CyberKnife QC program. Additionally, dedicated Farmer ionization chamber should be used to minimize detector-based errors and calibration of each detector should be also performed by secondary standard dosimetry laboratory (SSDL) at regular time intervals recommended by international reports. Furthermore, double cross-check should be performed with another calibrated ionization chamber and electrometer if unexpected measurement value is found in order to determine if deviation is due to ionization chamber, electrometer or other factors.

In addition to output stability, SRS/SRT facilities with CyberKnife require high degree of targeting accuracy or mechanical stability with respect to conventional linac-based treatment techniques such as 3-dimensional conformal radiotherapy (3DCRT), IMRT, and VMAT. As small positioning errors in SRS/SRT equipment can result in considerable changes to calculated dose of target volume and affect adjacent critical organs due to steep dose gradient and small target volume. For this reason, effective QC program should be implemented to ensure that the accuracy of the CyberKnife system does not deviate significantly from the baseline. AQA test is useful to check the spatial coordinate system of the manipulator. In addition, detailed analysis of AQA test results can provide valuable information about the short-term and long-term mechanical stability of the CyberKnife unit. In the pres- ent study, mechanical stability of CyberKnife system on yearly basis was analyzed and results indicated that pointing accuracy of Cyberknife system was well below the tolerance limit of $1 \mathrm{~mm}$, as recommended by AAPM TG-135, between the years of 2009 and 2011. However, 11 of the 98 measurements taken in 2012 and 2013 exceeded tolerance value; 7 were taken in May 2012 and remaining 4 were taken in November 2013. The manipulator, hence, was recalibrated only twice over 5 years. Although, AQA test provide valuable information about targeting accuracy, several parameters should be controlled before each calibration and E2E tests should be performed to ensure whether this deviation is caused by the robot or other factors like film scanner, film displacement and kilovoltage $(\mathrm{kV})$ imaging system.

Overall, analysis showed that output and mechanical stability of CyberKnife was very reliable over 5 years. Nevertheless, daily output measurements and AQA test are strongly recommended by the AAPM TG-135. AQA test is a quick test to evaluate the pointing accuracy of CyberKnife unit. However it has several limitations or disadvantages. The first is that it only provides information on translational parameters (left-right, superior-inferior and anterior-posterior) of the robotic system. Second, it is very costly and time-consuming to take daily measurements. Based on these limitations, AQA tests should be modified by the manufacturer to verify both translational and rotational parameters and a new simple module designed for a quick check of both mechanical and output stability of the system.

\section{Conclusion}

Output data of CyberKnife system is quite stable in daily measurements and in the long-term. In spite of its stability, daily measurements should be performed in SRS/SRT units like CyberKnife Robotic Radiosurgery System since high radiation dose of about 5-34 Gy/fr is usually delivered to target volume. It can be also pointed out that although 11 of 242 AQA tests exceeded the tolerance limit, long-term mechanical stability of the system is reliable, since maximum radial error was below $1.5 \mathrm{~mm}$ over 5 years.

\section{Acknowledgements}

This study was supported by Hacettepe University Scientific Research and Development Office Grant Project: 1-05 A 101009. 
Conflict of interest: None declared.

\section{References}

1. Sanghangthum T, Suriyapee S, Srisatit S, Pawlicki T. Retrospective analysis of linear accelerator output constancy checks using process control techniques. J Appl Clin Med Phys 2013;14(1):4032.

2. Kapanen M, Bly R, Sipilä P, Järvinen H, Tenhunen M. How can a cost/benefit ratio be optimized for an output measurement program of external photon radiotherapy beams? Phys Med Biol 2011;56(7):2119-30.

3. Huq MS, Fraass BA, Dunscombe PB, Gibbons JP Jr, Ibbott GS, Medin PM, et al. A method for evaluating quality assurance needs in radiation therapy. Int J Radiat Oncol Biol Phys 2008;71(1 Suppl):170-3.

4. Kapanen $M$, Tenhunen $M$, Hämäläinen $T$, Sipilä $P$, Parkkinen R, Järvinen H. Analysis of quality control data of eight modern radiotherapy linear accelerators: the short- and long-term behaviours of the outputs and the reproducibility of quality control measurements. Phys Med Biol 2006;51(14):3581-92.

5. Institution of Physics and Engineering in Medicine and Biology (IPEMB). Physical aspects of quality control in radiotherapy. IPEMB Report 81. New York: 1999.

6. Kutcher GJ, Coia L, Gillin M, Hanson WF, Leibel S, Morton RJ, et al. Comprehensive QA for radiation oncology: report of AAPM Radiation Therapy Committee Task Group 40. Med Phys 1994;21(4):581-618.

7. Netherlands Commission on Radiation Dosimetry (NCS). Quality control of medical linear accelerators: current practice and minimum requirements. NCS Report 9. Delft 1996.

8. Swiss Society of Radiobiology and Medical Physics (SSRMP/SGSMP). Quality control of medical electron accelerators. SSRMP Recommendations No.11. Bern 2003.

9. World Health Organisation (WHO). Quality Assurance in Radiotherapy. Geneva 1988.

10. Biggs PJ. Review of the energy check of an electron-only linear accelerator over a 6 year period: sensitivity of the technique to energy shift. Med Phys 2003;30(4):635-9.

11. Pawlicki T, Whitaker M, Boyer AL. Statistical process control for radiotherapy quality assurance. Med Phys 2005;32(9):2777-86.

12. Benneyan JC, Lloyd RC, Plsek PE. Statistical process control as a tool for research and healthcare improvement. Qual Saf Health Care 2003;12(6):458-64.

13. Noyez L. Control charts, Cusum techniques and funnel plots. A review of methods for monitoring performance in healthcare. Interact Cardiovasc Thorac Surg
2009;9(3):494-9.

14. Tennant R, Mohammed MA, Coleman JJ, Martin U. Monitoring patients using control charts: a systematic review. Int J Qual Health Care 2007;19(4):187-94.

15. Boggs PB, Wheeler D, Washburne WF, Hayati F. Peak expiratory flow rate control chart in asthma care: chart construction and use in asthma care. Ann Allergy Asthma Immunol 1998;81(6):552-62.

16. Holli K, Laippala P, Ojala A, Pitkänen M. Quality control in health care: an experiment in radiotherapy planning for breast cancer patients after mastectomy. Int J Radiat Oncol Biol Phys 1999;44(4):827-33.

17. Pawlicki T, Whitaker M. Variation and control of process behavior. Int J Radiat Oncol Biol Phys 2008;71(1 Suppl):210-4.

18. Breen SL, Moseley DJ, Zhang B, Sharpe MB. Statistical process control for IMRT dosimetric verification. Med Phys 2008;35(10):4417-25.

19. Pawlicki T, Yoo S, Court LE, McMillan SK, Rice RK, Russell JD, et al. Moving from IMRT QA measurements toward independent computer calculations using control charts. Radiother Oncol 2008;89(3):330-7.

20. Gérard K, Grandhaye JP, Marchesi V, Kafrouni H, Husson F, Aletti P. A comprehensive analysis of the IMRT dose delivery process using statistical process control (SPC). Med Phys 2009;36(4):1275-85.

21. Able CM, Hampton CJ, Baydush AH, Munley MT. Initial investigation using statistical process control for quality control of accelerator beam steering. Radiat Oncol 2011;6:180.

22. Nordström F, af Wetterstedt S, Johnsson S, Ceberg C, Bäck SJ. Control chart analysis of data from a multicenter monitor unit verification study. Radiother Oncol 2012;102(3):364-70.

23. Almond PR, Biggs PJ, Coursey BM, Hanson WF, Huq MS, Nath R, et al. AAPM's TG-51 protocol for clinical reference dosimetry of high-energy photon and electron beams. Med Phys 1999;26(9):1847-70.

24. Dieterich S, Cavedon C, Chuang CF, Cohen AB, Garrett JA, Lee CL, et al. Report of AAPM TG 135: quality assurance for robotic radiosurgery. Med Phys 2011;38(6):2914-36.

25. Sharma SC, Ott JT, Williams JB, Dickow D. Commissioning and acceptance testing of a CyberKnife linear accelerator. J Appl Clin Med Phys 2007;8(3):2473.

26. International Atomic Energy Agency. Absorbed dose determination in external beam radiotherapy. An International Code of Practice for Dosimetry based on standards of absorbed dose to water. Tech Rep Series No.398, IAEA. Vienna: 2000. 